\title{
$H$-MANIFOLDS HAVE NO NONTRIVIAL IDEMPOTENTS ${ }^{1}$
}

\section{ROBERT F. BROWN}

For a set $X$ and a function $m: X \times X \rightarrow X$, call $x \in X$ an idempotent (element) of $(X, m)$ if $m(x, x)=x$. If $(X, m)$ is a group with unit element $e$, then of course $e$, the trivial idempotent, is the only one in $(X, m)$. At the other extreme, if $m: X \times X \rightarrow X$ is defined by $m\left(x, x^{\prime}\right)$ $=x$, then $(X, m)$ is a semigroup in which every element is idempotent. We remark that the set of idempotents plays an important part in both the algebraic and topological theories of semigroups.

A triple $(X, m, e)$ is an $H$-space if $X$ is a topological space, $e \in X$, and $m: X \times X \rightarrow X$ is a map such that $m(x, e)=m(e, x)=x$ for all $x \in X$. This generalization of the group concept is certainly of as much interest in topology as that of semigroup. One is led, therefore, to ask whether an $H$-space is like a topological semigroup, with its rich theory of idempotents, or like a topological group, which has no nontrivial idempotents at all. The title of the paper gives the answer that, at least when the underlying space is a manifold, an $H$-space behaves much like a topological group in this respect.

One must, however, be careful in interpreting the statement above. For example, the interval $I=[0,1]$ is a manifold and $(I, m, 0)$ is an $H$-space when $m: I \times I \rightarrow I$ is defined by $m(s, t)=s t+|s-t|$ and yet $(I, m, 0)$ has a nontrivial idempotent. Observe that if we define, for $0 \leqq r \leqq 1$,

$$
m_{r}(s, t)=\frac{s t}{1+r}+|s-t|
$$

then $\left(I, m_{r}, 0\right)$ is an $H$-space. We thus have a "path" of $H$-spaces from the $H$-space $(I, m, 0)$ with a nontrivial idempotent to one without such an idempotent. We claim that this is the general situation. The point is that, unlike a group product, an $H$-space product is not thought of as a single function, but rather as an equivalence class of functions. With this interpretation, the statement in the title is correct.

Let $X$ be a space, $e \in X$, and let $H_{e}(X)$ denote the (possibly empty) space of maps $m: X \times X \rightarrow X$ such that $(X, m, e)$ is an $H$-space. Two elements of $H_{e}(X)$ are considered to be homotopic if they are in the same path component.

Received by the editors April 23, 1969.

1 The preparation of this paper was sponsored in part by NSF Grant GP-6530. 
Theorem. Let $(M, m, e)$ be an $H$-space where $M$ is a compact connected triangulable n-dimensional manifold with or without boundary, $n \geqq 3$. There exists $m^{\prime} \in H_{e}(M)$ homotopic to $m$ such that $\left(M, m^{\prime}, e\right)$ has no nontrivial idempotents.

The theorem contains new information even about Lie groups. For example, of course the two path components of $H_{c}\left(S^{3}\right)$ which contain group products contain maps without nontrivial idempotents, but now we know also that the other ten path components of $H_{e}\left(S^{3}\right)$ have the same property.

The proof of the theorem is an application of fixed point theory. Let $f: M \rightarrow M$ be a map such that $f(e)=e$. Fixed points $x$ and $x^{\prime}$ of $f$ are equivalent if there is a path $C: I \rightarrow M$ from $x$ to $x^{\prime}$ such that $C$ and $f C$ are homotopic by a homotopy which keeps $x$ and $x^{\prime}$ fixed. Write the abelian group $\pi_{1}(M, e)$ in additive notation and define $F_{*}: \pi_{1}(M, e)$ $\rightarrow \pi_{1}(M, e)$ by $F_{*}(\alpha)=f_{*}(\alpha)-\alpha$, where $f_{*}$ is the endomorphism of $\pi_{1}(M, e)$ induced by $f$. The number of equivalence classes of fixed points of $f$ is less than or equal to the order of the cokernel of $F_{*}[3$, Theorem 3.1].

Define $s: M \rightarrow M$ by $s(x)=m(x, x)$, then $s_{*}(\alpha)=2 \alpha$ for all $\alpha \in \pi_{1}(M, e)$ so $S_{*}$ is the identity function and consequently all the fixed points of $s$ are equivalent. Therefore, by Wecken's Theorem [5], there is a map $s^{\prime}: M \rightarrow M$ homotopic to $s$ such that $s^{\prime}$ has only a single fixed point. We will next examine a proof of Wecken's Theorem in order to show that we can choose $e$ to be the single fixed point of $s^{\prime}$ and that there is a homotopy $h_{t}: M \rightarrow M$ from $s$ to $s^{\prime}$ such that $h_{t}(e)=e$ for all $t \in I$.

Given $\epsilon>0$, there is a map $\bar{s}: M \rightarrow M$ with only a finite number of fixed points and a homotopy $s_{t}: M \rightarrow M$ such that $s_{0}=s, s_{1}=\bar{s}$, and for $d$ the metric of $M, d\left(s(x), s_{t}(x)\right)<\epsilon$ for all $x \in M$ and $t \in I$ [2]. Cover $M$ with a finite number of euclidean neighborhoods and take a triangulation of $M$ of mesh less than half the Lebesgue number of the cover. Choose the $\epsilon$ above small enough so that $e$ and $\bar{s}(e)$ lie in simplices of $M$ that have a common face. By Lemma 1.2 of [4], there is a homotopy $\bar{s}_{t}: M \rightarrow M$ such that $\bar{s}_{0}=\bar{s}$, the path $\left\{\bar{s}_{t}(e) \mid t \in I\right\}$ is contained in the union of the closed simplices of $M$ containing $e$ and $\bar{s}(e)$, and the fixed points of $\bar{s}_{1}$ consist of $e$ together with the fixed points of $\bar{s}$.

Observe that $s$ and $\bar{s}_{1}$ are homotopic by means of $s_{t}$ followed by $\bar{s}_{t}$. Furthermore, the loop $L_{e}$ consisting of the path $\left\{s_{t}(e) \mid t \in I\right\}$ followed by the path $\left\{\bar{s}_{t}(e) \mid t \in I\right\}$ is contained in an euclidean neighborhood and thus $L_{e}$ represents the unit element of $\pi_{1}(M, e)$. Conse- 
quently, by the homotopy extension theorem, there is a homotopy $\bar{h}_{t}: M \rightarrow M$ such that $\bar{h}_{0}=s, \bar{h}_{1}=\bar{s}_{1}$, and $\bar{h}_{t}(e)=e$ for all $t \in I$. The map $\bar{s}_{1}$ induces the same endomorphism of $\pi_{1}(M, e)$ as $s$ does, so all the fixed points of $\bar{s}_{1}$ are equivalent.

The proof of Theorem 2.4 of [4] tells us that there is a map $s^{*}: M$ $\rightarrow M$, homotopic to $\bar{s}_{1}$ by a homotopy keeping $e$ fixed, such that the only fixed points of $s^{*}$ are $e$ and another point $x_{0}$ in the same simplex of $M$. Of course $e$ and $x_{0}$ are equivalent fixed points of $s^{*}$. Furthermore, an argument within that same proof assures us that there is no loss of generality in assuming that the line segment from $e$ to $x_{0}$ and its image under $s^{*}$ are homotopic by a homotopy keeping $e$ and $x_{0}$ fixed. Therefore, we can apply Lemma 2.2 of [4] to obtain a map $s^{\prime}: M \rightarrow M$ whose only fixed point is $e$, and a homotopy $h_{t}^{\prime}$ from $s^{*}$ to $s^{\prime}$. An examination of the proof of Lemma 2.2 shows that $h_{t}^{\prime}(e)=e$ for all $t \in I$. We have proved that, given $s: M \rightarrow M$ defined by $s(x)$ $=m(x, x)$, there is indeed a map $s^{\prime}: M \rightarrow M$ with a single fixed point at $e$ and a homotopy $h_{t}: M \rightarrow M$ from $s$ to $s^{\prime}$ such that $h_{t}(e)=e$ for all $t \in I$.

Let $\Delta(M)=\{(x, x) \in M \times M\}$ and set

$$
A=(M \times\{e\}) \cup(\{e\} \times M) \cup \Delta(M) \subset M \times M \text {. }
$$

For

$$
T=(M \times M \times\{0\}) \cup(A \times I) \subset M \times M \times I,
$$

define $\mu: T \rightarrow M$ by

$$
\begin{aligned}
\mu(x, y, t) & =x & & \text { if } y=e, \\
& =y & & \text { if } x=e, \\
& =h_{t}(x) & & \text { if } x=y, \\
& =m(x, y) & & \text { if } t=0,
\end{aligned}
$$

then $\mu$ extends, by the homotopy extension theorem, to a map $\mu: M \times M \times I \rightarrow M$. Let $m^{\prime}: M \times M \rightarrow M$ be defined by $m^{\prime}(x, y)$ $=\mu(x, y, 1)$ then

$$
m^{\prime}(x, x)=h_{1}(x)=s^{\prime}(x) \neq x
$$

unless $x=e$, so we have proved the theorem.

The theorem can be proved for $H$-spaces $(X, m, e)$ where $X$ is a finite polyhedron of a type more general than a manifold essentially just by quoting the results of [4] in their full generality in the proof above. Although Wecken's Theorem is false for finite polyhedra in general, none of the known counterexamples support an $H$-space 
structure. Thus it may be that our theorem is true for all finite polyhedra. In any event, the first steps of the proof above do show that, given an $H$-space $(X, m, e)$ where $X$ is a connected finite polyhedron, there exists $m^{\prime} \in H_{e}(X)$ homotopic to $m$ such that $\left(X, m^{\prime}, e\right)$ has only a finite number of idempotents.

If one employs the techniques of [6] and [1] in place of those of [4], it is possible to prove the theorem for topological manifolds.

\section{REFERENCES}

1. R. F. Brown, On a homotopy converse to the Lefschetz fixed point theorem, Pacific J. Math. 17 (1966), 407-411. MR 33 \#3288.

2. H. Hopf, Über die algebraische Anzahl von Fixpunkten, Math. Z. 29 (1929), 493524.

3. Bo-ju Jiang, Estimation of the Nielsen numbers, Acta Math. Sinica 14 (1964), 304-312 = Chinese Math. Acta 5 (1964), 330-339. MR 30 \#1510.

4. Gen-hua Shi, On least number of fixed points and Nielson numbers, Acta. Math. Sinica 16 (1966), 223-232 = Chinese Math. Acta. 8 (1966), 234-243. MR 35 \#1004.

5. F. Wecken, Fixpunktklassen. III: Mindestzahlen von Fixpunkten, Math. Ann. 118 (1942), 544-577. MR 5, 275.

6. J. Weier, Fixpunkttheorie in topologischen Mannigfaltigkeiten, Math. Z. 59 (1953), 171-190. MR 15, 337.

University of California, Los Angeles 